\title{
APPROXIMATE FACTORIZATION CONSTRAINT PRECONDITIONERS FOR SADDLE-POINT MATRICES*
}

\author{
H. S. DOLLAR ${ }^{\dagger}$ AND A. J. WATHEN ${ }^{\dagger}$
}

\begin{abstract}
We consider the application of the conjugate gradient method to the solution of large, symmetric indefinite linear systems. Special emphasis is put on the use of constraint preconditioners and a new factorization that can reduce the number of flops required by the preconditioning step. Results concerning the eigenvalues of the preconditioned matrix and its minimum polynomial are given. Numerical experiments validate these conclusions.
\end{abstract}

Key words. preconditioning, indefinite linear systems, Krylov subspace methods, conjugate gradient method

AMS subject classifications. $65 \mathrm{~F} 10,65 \mathrm{~F} 50$

DOI. $10.1137 / 04060768 \mathrm{X}$

1. Introduction. There are many areas where we wish to solve matrix systems of the form

$$
\underbrace{\left[\begin{array}{cc}
A & B^{T} \\
B & 0
\end{array}\right]}_{\mathcal{A}}\left[\begin{array}{l}
x \\
y
\end{array}\right]=\underbrace{\left[\begin{array}{c}
c \\
d
\end{array}\right]}_{b},
$$

where $A \in \mathbb{R}^{n \times n}$ is symmetric and $B \in \mathbb{R}^{m \times n}$. We shall assume that $0<m \leq n, B$ is of full rank, and $A$ is positive definite in the nullspace of $B$. The symmetric matrix $\mathcal{A}$ is indefinite (i.e., it has both positive and negative eigenvalues.) Such systems may arise in convex quadratic programming problems [13] and saddle-point systems (see [2] and [10, Chapters 7, 9]), as well as many other areas.

Solution of systems of equations of the form (1.1) can be achieved by a number of methods. For large, sparse or structured matrices iterative methods are an attractive option. In particular, Krylov subspace methods apply techniques that involve orthogonal projections onto subspaces of the form

$$
\mathcal{K}(\mathcal{A}, b) \equiv \operatorname{span}\left\{b, \mathcal{A} b, \mathcal{A}^{2} b, \ldots, \mathcal{A}^{n-1} b, \ldots\right\}
$$

The conjugate gradient method (CG), minimum residual method (MINRES), and generalized minimal residual method (GMRES) are all common iterative Krylov subspace methods. The CG method is used for symmetric, positive definite matrices, MINRES for symmetric and possibly indefinite matrices, and GMRES for unsymmetric matrices [12].

MINRES and GMRES will find the solution of the linear system (1.1) within $n+m$ iterations in exact arithmetic, but CG may fail because of the indefiniteness of the system. GMRES is just an expensive way to implement MINRES when $\mathcal{A}$ is symmetric as here. For very large systems this upper bound on the number of

\footnotetext{
*Received by the editors May 3, 2004; accepted for publication (in revised form) December 21, 2004; published electronically February 3, 2006.

http://www.siam.org/journals/sisc/27-5/60768.html

${ }^{\dagger}$ Oxford University Computing Laboratory, Numerical Analysis Group, Wolfson Building, Parks Road, Oxford, OX1 3QD, UK (hsd@comlab.ox.ac.uk, wathen@comlab.ox.ac.uk). The work of the first author was supported by an EPSRC Doctoral Training Account.
} 
iterations is not helpful and such methods would generally not be employed unless many fewer iterations were required in practice; it is often advantageous to use a preconditioner $\mathcal{P}$ with such iterative methods. The preconditioner should reduce the number of iterations required for convergence but not significantly increase the amount of computation required at each iteration [12, Chapter 13].

Keller, Gould, and Wathen [8] investigated the use of a preconditioner of the form

$$
\mathcal{P}=\left[\begin{array}{cc}
G & B^{T} \\
B & 0
\end{array}\right]
$$

where $G$ approximates but is not the same as $A$. They were able to prove various results about the eigenvalues and eigenvectors for the preconditioned systems $\mathcal{P}^{-1} \mathcal{A}$, where $\mathcal{A}$ and $\mathcal{P}$ are defined in (1.1) and (1.2), respectively; $\mathcal{P}$ is called a constraint preconditioner.

The system (1.1) is symmetric and indefinite, implying that we are unable to apply the CG method directly in a robust way since it might fail [3, section 6.3]. Gould, Hribar, and Nocedal [7] show how the CG method can still be used when solving quadratic programming problems provided a preconditioner of the form (1.2) is employed. We extend their method to the general problem of solving systems of the form (1.1) where a constraint preconditioner of the form (1.2) is also used in sections 2 and 3 . In section 4 we introduce a new factorization for the preconditioner $\mathcal{P}$. This previously unpublished factorization was recently developed by Wil Schilders (Tech. Univ. Eindhoven/Philips) [11]. We shall refer to this as the Schilders factorization. In section 5 we give numerical examples to demonstrate the effectiveness of the use of this method. A related but different factorization is the variable-reduction method [4, Chapter 5].

2. CG method for the reduced system. We wish to solve the system (1.1) to find $\left[x^{* T} y^{* T}\right]^{T}$. Let $Z$ be an $n \times(n-m)$ matrix spanning the nullspace of $B$; then $B Z=0$. The columns of $B^{T}$ together with the columns of $Z$ span $\mathbb{R}^{n}$ and any solution $x^{*}$ of linear equations $B x=d$ can be written as

$$
x^{*}=B^{T} x_{B}^{*}+Z x_{Z}^{*} .
$$

Substituting this into (1.1) gives

$$
\left[\begin{array}{cc}
A & B^{T} \\
B & 0
\end{array}\right]\left[\begin{array}{c}
B^{T} x_{B}^{*}+Z x_{z}^{*} \\
y^{*}
\end{array}\right]=\left[\begin{array}{l}
c \\
d
\end{array}\right] .
$$

Let us split the matrix $\mathcal{A}$ into a block $3 \times 3$ structure where each corner block is of dimension $m \times m$. We can also expand the vector $\left[x^{* T} y^{* T}\right]^{T}$ into a matrix-vector product, $\mathcal{H} \rho^{*}$. Let $Z=\left[Z_{1}^{T} Z_{2}^{T}\right]^{T}$. Expression (2.2) then becomes

$$
\left[\begin{array}{ccc}
A_{1,1} & A_{1,2} & B_{1}^{T} \\
A_{2,1} & A_{2,2} & B_{2}^{T} \\
B_{1} & B_{2} & 0
\end{array}\right] \underbrace{\left[\begin{array}{ccc}
B_{1}^{T} & Z_{1} & 0 \\
B_{2}^{T} & Z_{2} & 0 \\
0 & 0 & I
\end{array}\right]}_{\mathcal{H}} \underbrace{\left[\begin{array}{c}
x_{B}^{*} \\
x_{Z}^{*} \\
y^{*}
\end{array}\right]}_{\rho^{*}}=\left[\begin{array}{c}
c_{1} \\
c_{2} \\
d
\end{array}\right] .
$$

To maintain symmetry of our system we premultiply (2.3) by $\mathcal{H}^{T}$. Multiplying the matrix expression $\mathcal{H}^{T} \mathcal{A H}$ and simplifying, we obtain the linear system

$$
\left[\begin{array}{ccc}
B A B^{T} & B A Z & B B^{T} \\
Z^{T} A B^{T} & Z^{T} A Z & 0 \\
B B^{T} & 0 & 0
\end{array}\right]\left[\begin{array}{c}
x_{B}^{*} \\
x_{z}^{*} \\
y^{*}
\end{array}\right]=\left[\begin{array}{c}
B c \\
Z^{T} c \\
d
\end{array}\right] .
$$


We observe that an $m \times m$ system determines $x_{B}^{*}$ :

$$
B B^{T} x_{B}^{*}=d .
$$

Since $B$ is of full rank, $B B^{T}$ is symmetric, positive definite. We could therefore solve this system using the Cholesky factorization of $B B^{T}$ if the dimension of the system is small enough [5, p. 143]. From (2.4), having found $x_{B}^{*}$, we can find $x_{z}^{*}$ by solving

$$
A_{z z} x_{z}^{*}=-c_{z}
$$

where

$$
A_{Z Z}=Z^{T} A Z, \quad c_{Z}=Z^{T}\left(A B^{T} x_{B}^{*}-c\right) .
$$

The matrix $A$ is symmetric, positive definite in the nullspace of $B$; hence $Z^{T} A Z$ is symmetric, positive definite. Anticipating our technique, we can apply the CG method to compute an approximate solution to the system (2.6). Substituting this into (2.1) will give us an approximate solution for $x^{*}$. Using (2.4) with (2.1) we obtain a system that can be solved to give an approximate solution for $y^{*}$ :

$$
B B^{T} y^{*}=B\left(c-A x^{*}\right) .
$$

If we used a Cholesky factorization to find $x_{B}^{*}$, then this same factorization could be employed to solve (2.7).

Let us consider the practical application of the CG method to the system (2.6). As we noted in section 1 , the use of preconditioning can improve the rate of convergence of the CG iteration. Let us assume that a preconditioner $W_{Z Z}$ is given, where $W_{Z Z}$ is a symmetric, positive definite matrix of dimension $n-m$. Let us consider the class of preconditioners of the form $W_{z Z}=Z^{T} G Z$, where $G$ is a symmetric matrix such that $Z^{T} G Z$ is positive definite. The preconditioned CG method can then be applied to the $(n-m)$-dimensional reduced system $A_{z z} x_{z}^{*}=-c_{z}$ (see [7, Algorithm 2.1]).

3. CG method for the full system. Explicit use of Algorithm 2.1 from [7] would require knowledge of $Z$ and solution of systems involving $W_{Z Z}$. The algorithm may, however, be written to make explicit use of the constraint preconditioner without the need for $Z$ at all. Let us define the scaled projection matrix

$$
P=Z\left(Z^{T} G Z\right)^{-1} Z^{T}
$$

We will later see that $P$ is independent of the choice of nullspace basis $Z$.

Algorithm 3.1 (preconditioned CG in expanded form). Choose an initial point $x$ satisfying $B x=d$, compute $r=A x-c, g=\operatorname{Pr}$, and $p=-g$. Repeat the following steps, until a convergence test is satisfied:

$$
\begin{aligned}
x & \leftarrow x+\alpha p, \text { where } \alpha=r^{T} g / p^{T} A p, \\
r^{+} & =r+\alpha A p, \\
g^{+} & =P r^{+}, \\
p & \leftarrow-g^{+}+\beta p, \text { where } \beta=\left(r^{+}\right)^{T} g^{+} / r^{T} g, \\
g & \leftarrow g^{+} \text {and } r \leftarrow r^{+} .
\end{aligned}
$$

Note the definition of $g^{+}$via the projection step $g^{+}=\mathrm{Pr}^{+}$. Following the terminology of [7], the vector $g^{+}$will be called the preconditioned residual. It is defined to 
be in the nullspace of $B$. We now wish to be able to apply the projection operator $Z\left(Z^{T} G Z\right)^{-1} Z^{T}$ without a representation of the nullspace basis $Z$. If $G$ is nonsingular, then $P$ can be expressed as

$$
P=G^{-1}\left(I-B^{T}\left(B G^{-1} B^{T}\right)^{-1} B G^{-1}\right) .
$$

We can find $g^{+}$by solving the system

$$
\left[\begin{array}{cc}
G & B^{T} \\
B & 0
\end{array}\right]\left[\begin{array}{l}
g^{+} \\
v^{+}
\end{array}\right]=\left[\begin{array}{c}
r^{+} \\
0
\end{array}\right]
$$

whenever $z^{T} G z \neq 0$ for all nonzero $z$ for which $B z=0$ [4, section 5.4.1]. The idea in Algorithm 3.2 below is to replace the projection step with the solution of (3.3) to define the same $g^{+}$: this is why a constraint preconditioner of the form (1.2) is needed. A preconditioner which is not of constraint form would not suffice.

Discrepancy in the magnitudes of $g^{+}$and $r^{+}$can cause numerical difficulties for which Gould, Hribar, and Nocedal [7] suggest using a residual update strategy that redefines $r^{+}$so that its norm is closer to that of $g^{+}$. This dramatically reduces the roundoff errors in the projection operation in practice.

Algorithm 3.2 (preconditioned CG with residual update (PCG)). Apply Algorithm 3.1 with the following changes:

Replace $g=\operatorname{Pr}$ by solving

$$
\left[\begin{array}{cc}
G & B^{T} \\
B & 0
\end{array}\right]\left[\begin{array}{l}
g \\
v
\end{array}\right]=\left[\begin{array}{l}
r \\
0
\end{array}\right]
$$

set $y=v$ and $r \leftarrow r-B^{T} y$ at the end of the initialization stage, replace $\mathrm{g}^{+}=\mathrm{Pr}^{+}$by solving (3.3),

replace $r \leftarrow r^{+}$by $r \leftarrow r^{+}-B^{T} v^{+}$.

The key point is that Algorithm 3.2 does not require the computation of any nullspace basis but is a CG procedure for a symmetric, positive definite system which yields the solution of the indefinite system (1.1).

We would therefore like to be able to solve the systems of the form (3.3) efficiently. If we were to use Gaussian elimination to solve this system then, in general, the number of flops required would be $\mathcal{O}\left((n+m)^{3}\right)$ [5, p. 98]. Although this may be reduced depending on the sparsity, it could be impractical for large systems. In the following section we introduce a different factorization for matrices of the form $\mathcal{P}$ in (1.2). If $2 m \leq n$, then we can use this factorization to solve $(3.3)$ in $\mathcal{O}\left((n-m)^{3}\right)$ flops. If $2 m>n$, then the number of flops required is $\mathcal{O}\left(\mathrm{m}^{3}\right)$.

4. Schilders' factorization for preconditioning step. The preconditioning step of solving (3.3) in Algorithm 3.2 involves a constraint preconditioner of the same form of $\mathcal{P}$ given in (1.2). Let us split $\mathcal{P}$ into a block $3 \times 3$ structure as we did for the matrix $\mathcal{A}$ in (2.3). Suppose we choose matrices $L_{1} \in \mathbb{R}^{m \times m}$ and $L_{2} \in \mathbb{R}^{(n-m) \times(n-m)}$ such that $L_{2}$ is nonsingular and assume that $B_{1}$ is nonsingular; then we can factorize $\mathcal{P}$ in the following manner:

$$
\mathcal{P}=\underbrace{\left[\begin{array}{ccc}
B_{1}{ }^{T} & 0 & L_{1} \\
B_{2}{ }^{T} & L_{2} & E \\
0 & 0 & I
\end{array}\right]}_{\mathcal{P}_{1}} \underbrace{\left[\begin{array}{ccc}
D_{1} & 0 & I \\
0 & D_{2} & 0 \\
I & 0 & 0
\end{array}\right]}_{\mathcal{P}_{2}} \underbrace{\left[\begin{array}{ccc}
B_{1} & B_{2} & 0 \\
0 & L_{2}{ }^{T} & 0 \\
L_{1}^{T} & E^{T} & I
\end{array}\right]}_{\mathcal{P}_{3}},
$$


where

$$
\begin{aligned}
D_{1} & =B_{1}{ }^{-T} G_{1,1} B_{1}{ }^{-1}-L_{1}{ }^{T} B_{1}{ }^{-1}-B_{1}{ }^{-T} L_{1}, \\
D_{2} & =L_{2}{ }^{-1}\left(G_{2,2}-B_{2}^{T} D_{1} B_{2}-E B_{2}-B_{2}^{T} E^{T}\right) L_{2}{ }^{-T}, \\
E & =G_{2,1} B_{1}^{-1}-B_{2}^{T} D_{1}-B_{2}^{T} L_{1}^{T} B_{1}^{-1} .
\end{aligned}
$$

We should emphasize that ensuring that $B_{1}$ is nonsingular by permutation of columns, if necessary, is an important and nontrivial practical issue.

Equations (4.2), (4.3), and (4.4) are such that we can choose the matrix $G$ and use this to define $D_{1}, D_{2}$, and $E$, or we could choose matrices $D_{1}, D_{2}$, and $E$ that then define the matrix $G$. If we choose to use the second option, then we need to make sure that $G$ satisfies several criteria set out in sections 2 and 3 . These are

- $G$ is nonsingular, and

- $Z^{T} G Z$ is symmetric, positive definite.

Since $\mathcal{P}_{1}\left(=\mathcal{P}_{3}^{T}\right)$ is nonsingular, $G$ will certainly satisfy these criteria if $\mathcal{P}_{2}$ is symmetric, positive definite. This is equivalent to the simple condition that $D_{2}$ is symmetric, positive definite.

In using the factorization (4.1) to solve (3.3), we take $L_{2}=I$ for simplicity. We then need to calculate the lu factorization of $B_{1} \in \mathbb{R}^{m \times m}$ and the Cholesky factorization of $D_{2} \in \mathbb{R}^{(n-m) \times(n-m)}$ once and then use these factored forms in each iteration. Note that we need only choose $D_{2}$ to be symmetric, positive definite, so we can effectively choose how much work is involved in the factorization of $D_{2}$ : the choice $D_{2}=I$ will give a less effective preconditioner but renders this Cholesky factorization trivial. If $i$ iterations are carried out in Algorithm 3.2, then there are $i+1$ solves carried out using the factorization (4.1). For general $B, D_{1}, D_{2}, E, L_{1}$, and $L_{2}$, the total number of flops required by PCG is given by

$$
\text { no. } \quad \text { flops. } \sim \frac{2}{3}\left(2(n-m)^{3}+m^{3}\right)+2(i+1)\left(13 m^{2}+3 n^{2}\right) .
$$

However, if $D_{2}$ and $L_{2}$ are diagonal, then the number of flops required reduces to

$$
\text { no. } \quad \text { flops. } \sim \frac{2}{3} m^{3}+4 m(i+1)(5 m+3 n) .
$$

We would like to balance this reduction in the number of flops with the possible increase in the number of iterations carried out.

Keller, Gould, and Wathen give a bound on the dimension of the Krylov subspace $\mathcal{K}\left(\mathcal{P}^{-1} \mathcal{A}, b\right)$. Proof of the following theorem can be found in [8].

TheOREM 4.1. Let $\mathcal{A} \in \mathbb{R}^{(n+m) \times(n+m)}$ be a symmetric and indefinite matrix of the form

$$
\mathcal{A}=\left[\begin{array}{cc}
A & B^{T} \\
B & 0
\end{array}\right]
$$

where $A \in \mathbb{R}^{n \times n}$ is symmetric and $B \in \mathbb{R}^{m \times n}$ is of full rank. Assume $Z$ is an $n \times(n-m)$ basis for the nullspace of $B$. Preconditioning $\mathcal{A}$ by a matrix of the form

$$
\mathcal{P}=\left[\begin{array}{cc}
G & B^{T} \\
B & 0
\end{array}\right]
$$

where $G \in \mathbb{R}^{n \times n}$ is symmetric, $G \neq A$, and $B \in \mathbb{R}^{m \times n}$ is as above, implies that 
- the matrix $\mathcal{P}^{-1} \mathcal{A}$ has

1. an eigenvalue at 1 with multiplicity $2 m$, and

2. $n-m$ eigenvalues $\lambda$ which are defined by the generalized eigenvalue problem $Z^{T} A Z x_{z}=\lambda Z^{T} G Z x_{z}$,

- the dimension of the Krylov subspace $\mathcal{K}\left(\mathcal{P}^{-1} \mathcal{A}, b\right)$ is at most $n-m+2$.

Let us consider what happens if $G_{1,1}=A_{1,1}, G_{1,2}=G_{2,1}{ }^{T}=A_{1,2}$, and we choose some matrix $D_{2}$ which is symmetric, positive definite. Equation (4.3) can be rearranged to give an explicit expression for $G_{2,2}$ : this is given in Theorem 4.2. We assume that $G_{2,2} \neq A_{2,2}$. By choosing $D_{2}$ and using the factorization (4.1) to solve (3.3), we will never have to explicitly form the matrix $\mathcal{P}$.

Theorem 4.1 reveals that the preconditioned system $\mathcal{P}^{-1} \mathcal{A}$ has an eigenvalue at 1 with multiplicity $2 m$, and $n-m$ eigenvalues which are defined by the generalized eigenvalue problem $Z^{T} A Z x_{z}=\lambda Z^{T} G Z x_{z}$. Noting that $B_{1}$ is nonsingular, we observe that $Z=\left[-B_{2}{ }^{T} B_{1}{ }^{-T} I\right]^{T} L_{2}{ }^{-T}$ is an $n \times(n-m)$ basis for the nullspace of $B$. Using the above values of $G_{1,1}, G_{1,2}, G_{2,1}$, and $G_{2,2}$, it is straightforward to show that the $n-m$ eigenvalues can be defined by

$$
\widehat{D}_{2} x_{z}=\lambda D_{2} x_{z}
$$

where

$$
\widehat{D}_{2}=L_{2}^{-1}\left(A_{2,2}-B_{2}^{T} D_{1} B_{2}-E B_{2}-B_{2}^{T} E^{T}\right) L_{2}{ }^{-T} .
$$

In the optimization community, $\widehat{D}_{2}$ is called the reduced Hessian.

Note that both $D_{2}$ and $\widehat{D}_{2}$ are symmetric, positive definite, so the eigenvalues are all real. Using the preconditioning matrix $\mathcal{P}$ with $G=\left[\begin{array}{lll}A_{1,1} & A_{1,2} ; A_{2,1} & G_{2,2}\end{array}\right]$, we can improve on the Krylov subspace dimension given in Theorem 4.1.

THEOREM 4.2. Let $\mathcal{A} \in \mathbb{R}^{(n+m) \times(n+m)}$ be a symmetric and indefinite matrix of the form

$$
\mathcal{A}=\left[\begin{array}{cc}
A & B^{T} \\
B & 0
\end{array}\right]
$$

where $A \in \mathbb{R}^{n \times n}$ is symmetric and $B \in \mathbb{R}^{m \times n}$ is of full rank with the first $m$ columns linearly dependent of each other. Let $A=\left[\begin{array}{llll}A_{1,1} & A_{1,2} ; & A_{2,1} & A_{2,2}\end{array}\right]$ and $B=\left[\begin{array}{ll}B_{1} & B_{2}\end{array}\right]$, where $A_{1,1}, B_{1} \in \mathbb{R}^{m \times m}, A_{1,2} \in \mathbb{R}^{m \times(n-m)}, A_{2,1}, B_{2} \in \mathbb{R}^{(n-m) \times m}$, and $A_{2,2} \in$ $\mathbb{R}^{(n-m) \times(n-m)}$.

Assume that $m<n$ and $\mathcal{A}$ is nonsingular. Let us choose any matrices $L_{1} \in$ $\mathbb{R}^{m \times m}, L_{2}, D_{2} \in \mathbb{R}^{(n-m) \times(n-m)}$ such that $L_{2}$ is nonsingular and $D_{2}$ is symmetric, positive definite. Assume $\mathcal{A}$ is preconditioned by a matrix of the form

$$
\mathcal{P}=\left[\begin{array}{ccc}
A_{1,1} & A_{1,2} & B_{1}^{T} \\
A_{2,1} & G_{2,2} & B_{2}^{T} \\
B_{1} & B_{2} & 0
\end{array}\right]
$$

where

$$
G_{2,2}=L_{2} D_{2} L_{2}{ }^{T}-B_{2}{ }^{T} B_{1}{ }^{-T} A_{1,1} B_{1}{ }^{-1} B_{2}+A_{2,1} B_{1}{ }^{-1} B_{2}+B_{2}{ }^{T} B_{1}{ }^{-T} A_{1,2} ;
$$

then the dimension of the Krylov subspace $\mathcal{K}\left(\mathcal{P}^{-1} \mathcal{A}, b\right)$ is at most $n-m+1$.

Proof. By assumption, $B_{1}$ is nonsingular so we can use Schilders' factorization (4.1) to express both $\mathcal{A}$ and $\mathcal{P}$. Using these factorizations we can express $\mathcal{P}^{-1} \mathcal{A}$ as

$$
\mathcal{P}^{-1} \mathcal{A}=\left[\begin{array}{ccc}
I & \Theta & 0 \\
0 & L_{2}{ }^{-T} D_{2}{ }^{-1} \widehat{D}_{2} L_{2}{ }^{T} & 0 \\
0 & \Upsilon & I
\end{array}\right],
$$


where $D_{1}, \widehat{D}_{2}$, and $E$ are given by (4.2), (4.6), and (4.4), respectively, and

$$
\begin{aligned}
\Theta & =B_{1}{ }^{-1} B_{2}\left(I-L_{2}{ }^{-T} D_{2}{ }^{-1} \widehat{D}_{2} L_{2}{ }^{T}\right), \\
\Upsilon & =\left(E^{T}-L_{1}{ }^{T} B_{1}{ }^{-1} B_{2}\right)\left(I-L_{2}{ }^{-T} D_{2}{ }^{-1} \widehat{D}_{2} L_{2}{ }^{T}\right) .
\end{aligned}
$$

From the eigenvalues of the preconditioned system, it is evident that the characteristic polynomial of the preconditioned system is of the form

$$
\left(\mathcal{P}^{-1} \mathcal{A}-I\right)^{2 m} \prod_{i=1}^{n-m}\left(\mathcal{P}^{-1} \mathcal{A}-\lambda_{i} I\right)
$$

To prove the upper bound on the dimension of the Krylov subspace we need to show that the degree of the minimum polynomial is less than or equal to $n-m+1$. Expanding the polynomial $\prod_{i=1}^{n-m}\left(\mathcal{P}^{-1} \mathcal{A}-\lambda_{i} I\right)$ of degree $n-m$, we obtain a matrix of the form

$$
\left[\begin{array}{ccc}
\prod_{i=1}^{n-m}\left(1-\lambda_{i}\right) I & f_{n-m}(\Theta) & 0 \\
0 & \prod_{i=1}^{n-m}\left(S-\lambda_{i} I\right) & 0 \\
0 & f_{n-m}(\Upsilon) & \prod_{i=1}^{n-m}\left(1-\lambda_{i}\right) I
\end{array}\right]
$$

where $S=L_{2}{ }^{-T} D_{2}{ }^{-1} \widehat{D}_{2} L_{2}{ }^{T}$ and $f_{n-m}(\cdot)$ is defined by the recursive formula

$$
f_{n-m}(\Gamma)=\Gamma \prod_{i=1}^{n-m-1}\left(1-\lambda_{i}\right) I+f_{n-m-1}(\Gamma)\left(S-\lambda_{n-m} I\right)
$$

with base case $f_{1}(\Gamma)=\Gamma$. Now let us premultiply the matrix (4.10) by $\mathcal{P}^{-1} \mathcal{A}-I$ to give

$$
\left[\begin{array}{ccc}
0 & \Theta \prod_{i=1}^{n-m}\left(S-\lambda_{i} I\right) & 0 \\
0 & (S-I) \prod_{i=1}^{n-m}\left(S-\lambda_{i} I\right) & 0 \\
0 & \Upsilon \prod_{i=1}^{n-m}\left(S-\lambda_{i} I\right) & 0
\end{array}\right]
$$

We note that the $(1,2),(2,2)$, and $(3,2)$ entries are in fact zero, since the $\lambda_{i}(i=$ $1, \ldots, n-m)$ are eigenvalues of $S$, which is similar to a symmetric matrix and thus diagonalizable. Hence, the degree of the minimum polynomial of $\mathcal{P}^{-1} \mathcal{A}$ is less than or equal to $n-m+1$.

If at least one of the eigenvalues defined by (4.5) has multiplicity greater than one, then we can sharpen this bound.

TheOREM 4.3. Suppose we precondition our system $\mathcal{A}$ by a matrix $\mathcal{P}$ as defined in Theorem 4.2. Assume that there exists $\alpha \in \mathbb{R}$ and $1 \leq j<n-m$ such that the eigenvalues defined by (4.5) can be "ordered" to satisfy

$$
\begin{aligned}
& \lambda_{i} \neq \alpha, \quad i=1, \ldots, j, \\
& \lambda_{i}=\alpha \quad i=j+1, \ldots, n-m ;
\end{aligned}
$$

then the dimension of the Krylov subspace $\mathcal{K}\left(\mathcal{P}^{-1} \mathcal{A}, b\right)$ is at most $j+2$.

Proof. Following the proof of Theorem 4.2 we can show that

$$
\prod_{i=1}^{j+1}\left(\mathcal{P}^{-1} \mathcal{A}-\lambda_{i} I\right)=\left[\begin{array}{ccc}
\prod_{i=1}^{j+1}\left(1-\lambda_{i}\right) I & f_{j+1}(\Theta) & 0 \\
0 & \prod_{i=1}^{j+1}\left(S-\lambda_{i} I\right) & 0 \\
0 & f_{j+1}(\Upsilon) & \prod_{i=1}^{j+1}\left(1-\lambda_{i}\right) I
\end{array}\right]
$$


where $\Theta, \Upsilon, S$, and $f_{j+1}(\cdot)$ are as defined previously. Now let us premultiply the matrix (4.12) by $\mathcal{P}^{-1} \mathcal{A}-I$ to give

$$
\left[\begin{array}{ccc}
0 & \Theta \prod_{i=1}^{j+1}\left(S-\lambda_{i} I\right) & 0 \\
0 & (S-I) \prod_{i=1}^{j+1}\left(S-\lambda_{i} I\right) & 0 \\
0 & \Upsilon \prod_{i=1}^{j+1}\left(S-\lambda_{i} I\right) & 0
\end{array}\right]
$$

As in the proof of Theorem 4.2, we note that the $(1,2),(2,2)$, and $(3,2)$ entries are in fact zero. Hence, the degree of the minimum polynomial of $\mathcal{P}^{-1} \mathcal{A}$ is less than or equal to $j+2$.

TheOREm 4.4. Suppose we precondition our system $\mathcal{A}$ by a matrix $\mathcal{P}$ as defined in Theorem 4.2. Assume that the matrix $A_{2,2}$ is symmetric, positive definite. Let us define $D_{2}=L_{2}^{-1} A_{2,2} L_{2}^{-T}$; then the Krylov subspace $\mathcal{K}\left(\mathcal{P}^{-1} \mathcal{A}, b\right)$ has dimension at most $\min (2 m+2, n-m+1)$.

Proof. Let us expand the difference $\widehat{D_{2}}-D_{2}$ :

$$
\begin{aligned}
\widehat{D_{2}}-D_{2}= & L_{2}^{-1}\left(A_{2,2}+B_{2}^{T} B_{1}^{-T} A_{1,1} B_{1}^{-1} B_{2}-A_{2,1} B_{1}^{-1} B_{2}-B_{2}^{T} B_{1}^{-T} A_{1,2}\right) L_{2}^{-T} \\
& -L_{2}^{-1} A_{2,2} L_{2}^{-T} \\
= & L_{2}^{-1}\left(B_{2}^{T} B_{1}^{-T}\left(A_{1,1} B_{1}^{-1} B_{2}-A_{1,2}\right)-A_{2,1} B_{1}^{-1} B_{2}\right) L_{2}^{-T} .
\end{aligned}
$$

Now,

$$
\begin{aligned}
\operatorname{rank}\left(\widehat{D_{2}}-D_{2}\right) \leq & \min \left[\min \left[\operatorname{rank}\left(B_{2}\right), \min \left[\operatorname{rank}\left(A_{1,1}\right), \operatorname{rank}\left(B_{2}\right)\right]+\operatorname{rank}\left(A_{1,2}\right)\right]\right. \\
& \left.+\min \left[\operatorname{rank}\left(A_{2,1}\right), \operatorname{rank}\left(B_{2}\right)\right], n-m\right] \\
\leq & \min (2 m, n-m) .
\end{aligned}
$$

Since $D_{2}$ is, by assumption, positive definite, we may write $D_{2}=W W^{T}$ for some nonsingular $W$. Thus

$$
W^{-1} \widehat{D_{2}} W^{-T}=I+W^{-1} L_{2}^{-1}\left(B_{2}^{T} B_{1}^{-T}\left(A_{1,1} B_{1}^{-1} B_{2}-A_{1,2}\right)-A_{2,1} B_{1}^{-1} B_{2}\right) L_{2}^{-T} W^{-T}
$$

differs from the identity matrix by a matrix of rank at most $\min (2 m, n-m)$, and hence there are at most $\min (2 m, n-m)$ nonunit eigenvalues defined by the generalized eigenvalue problem (4.5). We can therefore arrange the eigenvalues such that there exists $1 \leq j \leq \min (2 m, n-m)$ and

$$
\begin{aligned}
& \lambda_{i} \neq 1, \quad i=1, \ldots, j, \\
& \lambda_{i}=1, \quad i=j+1, \ldots, n-m .
\end{aligned}
$$

From Theorems 4.2 and 4.3 we conclude that the dimension of the Krylov subspace $\mathcal{K}\left(\mathcal{P}^{-1} \mathcal{A}, b\right)$ is at most $\min (2 m+2, n-m+1)$.

5. Numerical examples. We apply Algorithm 3.2 to solve a selection of problems from the CUTEr collection [6], and also a set of Stokes saddle-point problems generated using the ifiss incompressible flow software associated with the book [2]. Any simple bounds in the CUTEr problems are removed to create a problem of the form

$$
\begin{aligned}
& \min _{x} f(x)=\frac{1}{2} x^{T} A x+s^{T} x \\
& \quad \text { subject to } B x=t .
\end{aligned}
$$

For both sets of problems, the matrix $A \in \mathbb{R}^{n \times n}$ is symmetric and $B \in \mathbb{R}^{m \times n}$ is the full row rank matrix of linear constraints; $A$ is positive definite in the nullspace 
TABLE 5.1

Values of $m, n$, nonzeros in $A$, and nonzeros in $B$ for the test problems.

\begin{tabular}{l|rr|rr} 
Problem & $m$ & $n$ & $\mathrm{nz}(A)$ & $\mathrm{nz}(B)$ \\
\hline CONT-050 & 2401 & 2597 & 2597 & 12005 \\
CONT-101 & 10098 & 10197 & 2700 & 49599 \\
CONT-300 & 90298 & 90597 & 90597 & 448799 \\
CONT1-10 & 9801 & 10197 & 10197 & 49005 \\
CVXQP1 & 5000 & 10000 & 69968 & 14998 \\
CVXQP2 & 2500 & 10000 & 69968 & 7499 \\
CVXQP3 & 7500 & 10000 & 69968 & 22497 \\
DUAL1 & 1 & 85 & 7031 & 85 \\
DUAL2 & 1 & 96 & 8920 & 96 \\
DUAL3 & 1 & 111 & 12105 & 111 \\
DUAL4 & 1 & 75 & 5523 & 75 \\
KSIP & 1021 & 1001 & 20 & 21002 \\
MOSARQP1 & 3200 & 700 & 2590 & 4122 \\
PRIMALC1 & 9 & 239 & 229 & 2079 \\
PRIMALC2 & 7 & 238 & 230 & 1624 \\
PRIMALC5 & 8 & 295 & 286 & 2304 \\
PRIMALC8 & 8 & 528 & 519 & 4168 \\
\hline STOKES1 & 288 & 2178 & 28418 & 10452 \\
STOKES2 & 1088 & 8450 & 122206 & 44306 \\
STOKES3 & 769 & 5890 & 82582 & 30483 \\
STOKES4 & 2945 & 23042 & 345234 & 126799 \\
STOKES5 & 2112 & 5890 & 82582 & 30064 \\
STOKES6 & 8448 & 23042 & 345234 & 129124 \\
STOKES7 & 255 & 2178 & 28568 & 3826 \\
STOKES8 & 1023 & 8450 & 122506 & 16771
\end{tabular}

of $B$ (in fact on the whole space for the Stokes problems), and vectors $x, s$, and $t$ have appropriate dimensions. A permuted lu factorization of $B^{T}$ is used to find a permutation matrix, $M$, such that the first $m$ columns of $B M$ are linearly independent [5, Chapter 3]. We then set $B \leftarrow B M, A \leftarrow M^{T} A M, s \leftarrow M^{T} s$, and $x \leftarrow M^{T} x$. The assumption about the nonsingularity of $B_{1}$ will now hold.

The dimensions of the problems and number of nonzeros in the associated matrices $A$ and $B$ are given in Table 5.1. Tables 5.2-5.6 show the results of using Algorithm 3.2 with different preconditioners and a low tolerance. The results of using the preconditioner $\mathcal{P}=\left[\begin{array}{ll}I B^{T} ; B & 0]\end{array}\right]$ are shown in Table 5.2. This preconditioner is of the general form considered in [8] (see also [9]). Another possible choice for $G$ is to use the diagonal entries of $A$ [1]. The results of using this preconditioner are shown in Table 5.3. In Tables 5.4 and 5.5 we use preconditioners of the form considered in Theorems 4.2 and 4.4 , respectively. The final preconditioner that we try will generally reproduce less of the matrix $A$ in $G$ than the previous two do, but we hope that this will be compensated by the iterations being "cheaper" to apply. The results are given in Table 5.6.

We compare the number of iterations and the amount of CPU time taken when we use an $\mathrm{lu}$ factorization of the preconditioner $\mathcal{P}$, and when we use the Schilders' factorization for $\mathcal{P}$. In both cases, we carry out the factorization once and then use the factored forms in each iteration. When producing Schilders' factorization, we set $L_{1}=0$ and $L_{2}=I$. At present, we have not carried out substantial research into how we should choose the values of $L_{1}$ and $L_{2}$. In an attempt to maintain sparsity, an approximate minimum degree preordering strategy is applied before carrying out any $\mathrm{lu}$ factorizations. Threshold pivoting is also used during the lu factorization stages: the default threshold value in MATLAB is used. 
TABLE 5.2

Solution statistics: Comparisons between iterative PCG solvers when using lu and Schilders' factorizations in the preconditioning step with tol $=10^{-2}, G=I$.

\begin{tabular}{l|rrrr|rrrr|r} 
& \multicolumn{6}{|c|}{ LU } & \multicolumn{5}{c|}{ Schilders' } & \\
Problem & Its & FTime & ITime & Total & Its & FTime & ITime & Total & Digits \\
\hline CONT-050 & 1 & 1.18 & 0.76 & 2.03 & 1 & 6.95 & 0.45 & 7.49 & 13 \\
CONT-101 & 1 & 21.43 & 6.53 & 28.73 & 1 & 123.38 & 2.91 & 127.06 & 9 \\
CONT-300 & - & - & - & - & - & - & - & - & - \\
CONT1-10 & 1 & 20.59 & 6.42 & 27.80 & 1 & 128.72 & 2.89 & 132.41 & 13 \\
CVXQP1 & 2 & 1.93 & 1.36 & 3.40 & 2 & 74.49 & 2.16 & 76.75 & 14 \\
CVXQP2 & 2 & 0.04 & 0.11 & 0.17 & 2 & 0.10 & 0.13 & 0.24 & 15 \\
CVXQP3 & 2 & 3.19 & 3.10 & 6.63 & 2 & 23.96 & 1.02 & 25.32 & 15 \\
DUAL1 & 5 & 0.00 & 0.00 & 0.00 & 5 & 0.01 & 0.01 & 0.01 & 15 \\
DUAL2 & 4 & 0.00 & 0.00 & 0.01 & 4 & 0.01 & 0.01 & 0.02 & 13 \\
DUAL3 & 5 & 0.00 & 0.01 & 0.01 & 5 & 0.01 & 0.01 & 0.02 & 14 \\
DUAL4 & 4 & 0.00 & 0.00 & 0.00 & 4 & 0.01 & 0.00 & 0.01 & 15 \\
KSIP & 17 & 0.34 & 0.91 & 2.11 & 18 & 0.03 & 0.87 & 1.63 & 5 \\
MOSARQP1 & 4 & 0.03 & 0.06 & 0.11 & 4 & 0.08 & 0.07 & 0.17 & 15 \\
PRIMALC1 & 2 & 0.00 & 0.01 & 0.01 & 2 & 0.08 & 0.02 & 0.10 & 12 \\
PRIMALC2 & 1 & 0.00 & 0.00 & 0.01 & 1 & 0.07 & 0.02 & 0.09 & 14 \\
PRIMALC5 & 0 & 0.00 & 2.12 & 2.13 & 0 & 0.16 & 22.07 & 22.23 & 15 \\
PRIMALC8 & 2 & 0.01 & 7.76 & 7.77 & 2 & 0.61 & 153.51 & 154.13 & 14 \\
\hline STOKES1 & 7 & 0.05 & 0.07 & 0.13 & 7 & 15.04 & 3.68 & 18.73 & 14 \\
STOKES2 & 8 & 0.27 & 0.34 & 0.69 & 8 & 932.91 & 116.33 & 1049.33 & 13 \\
STOKES3 & 2 & 0.26 & 0.12 & 0.44 & 2 & 280.59 & 16.54 & 297.18 & 11 \\
STOKES4 & 2 & 0.95 & 0.56 & 1.83 & - & - & - & - & -13 \\
STOKES5 & 2 & 0.20 & 0.26 & 0.62 & 2 & 69.71 & 6.01 & 75.87 & 12 \\
STOKES6 & 2 & 1.29 & 1.54 & 3.84 & - & - & - & - & -10 \\
STOKES7 & 9 & 0.02 & 0.07 & 0.10 & 9 & 10.48 & 0.32 & 10.80 & 14 \\
STOKES8 & 10 & 0.09 & 0.40 & 0.51 & 10 & 662.66 & 5.04 & 667.73 & 13
\end{tabular}

\begin{tabular}{l|rrr|rrr} 
& \multicolumn{3}{|c}{$\mathrm{LU}$} & \multicolumn{3}{c}{ Schilders' } \\
Problem & $\mathrm{nz}(L(\mathcal{P}))$ & $\mathrm{nz}(U(\mathcal{P}))$ & Total & $\mathrm{nz}\left(L\left(D_{2}\right)\right)$ & $\mathrm{nz}\left(U\left(D_{2}\right)\right)$ & Total \\
\hline CONT-050 & 630887 & 607170 & 1238057 & 19306 & 19306 & 155305 \\
CONT-101 & 5263734 & 6407090 & 11670824 & 4950 & 4950 & 700946 \\
CONT-300 & - & - & - & - & - & - \\
CONT1-10 & 5195636 & 6075386 & 11271022 & 78606 & 78606 & 833857 \\
CVXQP1 & 708449 & 801586 & 1510035 & 1229895 & 1241503 & 2519154 \\
CVXQP2 & 22168 & 25467 & 47635 & 17038 & 17038 & 46791 \\
CVXQP3 & 1682000 & 1267180 & 2949180 & 257477 & 259336 & 578439 \\
DUAL1 & 171 & 171 & 342 & 3570 & 3570 & 7227 \\
DUAL2 & 193 & 193 & 386 & 4560 & 4560 & 9218 \\
DUAL3 & 223 & 223 & 446 & 6105 & 6105 & 12323 \\
DUAL4 & 151 & 151 & 302 & 2775 & 2775 & 5627 \\
KSIP & 23214 & 23214 & 46428 & 210 & 210 & 23424 \\
MOSARQP1 & 24109 & 26831 & 50940 & 32887 & 32887 & 71296 \\
PRIMALC1 & 2363 & 4424 & 6787 & 26565 & 26565 & 55227 \\
PRIMALC2 & 1890 & 3500 & 5390 & 26796 & 26796 & 55230 \\
PRIMALC5 & 2635 & 4923 & 7558 & 41321 & 41328 & 84969 \\
PRIMALC8 & 4732 & 8884 & 13616 & 135460 & 135460 & 275104 \\
\hline STOKES1 & 21079 & 21079 & 42158 & 1335628 & 1334125 & 2683050 \\
STOKES2 & 105503 & 105503 & 211006 & 23447464 & 23461548 & 46971065 \\
STOKES3 & 63029 & 63029 & 126058 & 10908746 & 10877987 & 21825155 \\
STOKES4 & 326595 & 326595 & 653190 & - & - & - \\
STOKES5 & 135816 & 136414 & 272230 & 4010012 & 4050659 & 8095603 \\
STOKES6 & 746422 & 745095 & 1491517 & - & - & - \\
STOKES7 & 9155 & 9154 & 18309 & 1036995 & 1033457 & 2074914 \\
STOKES8 & 44741 & 44741 & 89482 & 20095193 & 20083780 & 40198321
\end{tabular}


TABLE 5.3

Solution statistics: Comparisons between iterative PCG solvers when using lu and Schilders' factorizations in the preconditioning step with tol $=10^{-2}, G=\operatorname{diag}(A)$.

\begin{tabular}{l|rrrr|rrrr|r} 
& \multicolumn{6}{|c|}{ LU } & \multicolumn{5}{c|}{ Schilders' } & \\
Problem & Its & FTime & ITime & Total & Its & FTime & ITime & Total & Digits \\
\hline CONT-050 & 1 & 1.28 & 0.74 & 2.12 & 1 & 6.98 & 0.45 & 7.53 & 13 \\
CONT-101 & 1 & 10.38 & 5.17 & 16.33 & 1 & 32.47 & 2.84 & 36.09 & 10 \\
CONT-300 & - & - & - & - & - & - & - & - & - \\
CONT1-10 & 1 & 18.70 & 6.28 & 25.78 & 1 & 128.01 & 2.92 & 131.71 & 13 \\
CVXQP1 & 1 & 1.61 & 0.93 & 2.64 & 1 & 74.61 & 1.47 & 76.18 & 11 \\
CVXQP2 & 1 & 0.04 & 0.08 & 0.14 & 1 & 0.10 & 0.10 & 0.22 & 15 \\
CVXQP3 & 1 & 3.45 & 2.40 & 6.20 & 1 & 23.14 & 0.80 & 24.28 & 13 \\
DUAL1 & 5 & 0.00 & 0.00 & 0.00 & 5 & 0.01 & 0.00 & 0.01 & 12 \\
DUAL2 & 4 & 0.00 & 0.00 & 0.01 & 4 & 0.01 & 0.01 & 0.02 & 14 \\
DUAL3 & 5 & 0.00 & 0.01 & 0.01 & 5 & 0.01 & 0.01 & 0.02 & 13 \\
DUAL4 & 4 & 0.00 & 0.00 & 0.00 & 4 & 0.01 & 0.00 & 0.01 & 13 \\
KSIP & 1 & 0.34 & 0.73 & 1.92 & 1 & 0.01 & 0.73 & 1.46 & 15 \\
MOSARQP1 & 2 & 0.02 & 0.03 & 0.07 & 2 & 0.02 & 0.04 & 0.08 & 15 \\
PRIMALC1 & 0 & 0.00 & 4.52 & 4.52 & 0 & 0.00 & 1.15 & 1.16 & 15 \\
PRIMALC2 & 0 & 0.00 & 4.23 & 4.24 & 0 & 0.00 & 1.00 & 1.01 & 15 \\
PRIMALC5 & 0 & 0.00 & 6.87 & 6.88 & 0 & 0.00 & 1.63 & 1.63 & 15 \\
PRIMALC8 & 0 & 0.01 & 28.54 & 28.55 & 0 & 0.00 & 5.15 & 5.15 & 15 \\
\hline STOKES1 & 5 & 0.05 & 0.05 & 0.12 & 5 & 14.79 & 3.75 & 18.56 & 14 \\
STOKES2 & 5 & 0.27 & 0.25 & 0.61 & 5 & 926.89 & 77.75 & 1004.72 & 15 \\
STOKES3 & 2 & 0.34 & 0.12 & 0.54 & 2 & 284.06 & 17.13 & 301.24 & 12 \\
STOKES4 & 2 & 0.88 & 0.55 & 1.74 & - & - & - & - & - \\
STOKES5 & 2 & 0.21 & 0.27 & 0.63 & 2 & 67.73 & 6.54 & 74.43 & 12 \\
STOKES6 & 2 & 1.30 & 1.55 & 3.85 & - & - & - & -16 & -15 \\
STOKES7 & 4 & 0.02 & 0.04 & 0.06 & 4 & 10.51 & 0.16 & 10.67 & 15 \\
STOKES8 & 4 & 0.09 & 0.19 & 0.31 & 4 & 663.26 & 2.35 & 665.64 & 13
\end{tabular}

\begin{tabular}{l|rrr|rrr} 
& \multicolumn{3}{|c}{$\mathrm{LU}$} & \multicolumn{3}{c}{ Schilders' } \\
Problem & $\mathrm{nz}(L(\mathcal{P}))$ & $\mathrm{nz}(U(\mathcal{P}))$ & Total & $\mathrm{nz}\left(L\left(D_{2}\right)\right)$ & $\mathrm{nz}\left(U\left(D_{2}\right)\right)$ & Total \\
\hline CONT-050 & 620089 & 639618 & 1259707 & 19306 & 19306 & 155305 \\
CONT-101 & 4255279 & 2928002 & 7183281 & 4950 & 4950 & 693449 \\
CONT-300 & - & - & - & - & - & - \\
CONT1-10 & 5135085 & 5655789 & 10790874 & 78606 & 78606 & 833857 \\
CVXQP1 & 723131 & 577861 & 1300992 & 1228384 & 1232830 & 2508970 \\
CVXQP2 & 21880 & 23198 & 45078 & 16921 & 16713 & 46349 \\
CVXQP3 & 1901464 & 1069000 & 2970464 & 257803 & 259757 & 579186 \\
DUAL1 & 171 & 171 & 342 & 3570 & 3570 & 7227 \\
DUAL2 & 193 & 193 & 386 & 4560 & 4560 & 9218 \\
DUAL3 & 223 & 223 & 446 & 6105 & 6105 & 12323 \\
DUAL4 & 151 & 151 & 302 & 2775 & 2775 & 5627 \\
KSIP & 22022 & 22024 & 44046 & 210 & 210 & 22424 \\
MOSARQP1 & 7289 & 7380 & 14669 & 5145 & 5145 & 15462 \\
PRIMALC1 & 248 & 4387 & 4635 & 230 & 229 & 2547 \\
PRIMALC2 & 245 & 3478 & 3723 & 231 & 230 & 2092 \\
PRIMALC5 & 303 & 4894 & 5197 & 287 & 286 & 2885 \\
PRIMALC8 & 536 & 8855 & 9391 & 520 & 519 & 5215 \\
\hline STOKES1 & 21079 & 21079 & 42158 & 1335797 & 1334073 & 2683167 \\
STOKES2 & 105503 & 105503 & 211006 & 23447428 & 23461508 & 46970989 \\
STOKES3 & 63029 & 63029 & 126058 & 10908667 & 10878627 & 21825716 \\
STOKES4 & 326595 & 326595 & 653190 & - & - & - \\
STOKES5 & 135821 & 136395 & 272216 & 3972246 & 4017853 & 8025031 \\
STOKES6 & 747054 & 745028 & 1492082 & - & -1035774 & 2079820 \\
STOKES7 & 9155 & 9154 & 18309 & 1039584 & 10359 \\
STOKES8 & 44741 & 44741 & 89482 & 20085901 & 20077317 & 40182566
\end{tabular}


TABLE 5.4

Solution statistics: Comparisons between iterative PCG solvers when using lu and Schilders' factorizations in the preconditioning step with tol $=10^{-2}, G_{1,1}=A_{1,1}, G_{1,2}=A_{1,2}$, and $D_{2}=$ $\operatorname{diag}\left(\widehat{D}_{2}\right)$.

\begin{tabular}{l|rrrr|rrrr|r} 
& \multicolumn{9}{|c}{ LU } \\
Problem & Its & FTime & ITime & Total & Its & FTime & ITime & Total & Digits \\
\hline CONT-050 & 1 & 9.63 & 0.66 & 10.39 & 1 & 6.98 & 0.44 & 7.51 & 12 \\
CONT-101 & 1 & 43.41 & 4.52 & 48.69 & 1 & 32.57 & 2.87 & 36.22 & 12 \\
CONT-300 & - & - & - & - & - & - & - & - & - \\
CONT1-10 & 1 & 159.98 & 5.70 & 166.48 & 1 & 128.90 & 2.91 & 132.61 & 12 \\
CVXQP1 & 12 & 743.04 & 245.24 & 988.39 & 11 & 33.01 & 1.29 & 34.40 & 11 \\
CVXQP2 & 3 & 125.36 & 22.92 & 148.29 & 3 & 0.56 & 0.15 & 0.72 & 14 \\
CVXQP3 & 5 & 644.13 & 105.32 & 749.79 & 5 & 24.08 & 1.07 & 25.50 & 6 \\
DUAL1 & 6 & 0.01 & 0.01 & 0.01 & 6 & 0.00 & 0.01 & 0.01 & 8 \\
DUAL2 & 5 & 0.02 & 0.01 & 0.03 & 5 & 0.01 & 0.01 & 0.01 & 8 \\
DUAL3 & 6 & 0.03 & 0.02 & 0.04 & 6 & 0.01 & 0.01 & 0.02 & 8 \\
DUAL4 & 4 & 0.01 & 0.00 & 0.02 & 4 & 0.01 & 0.00 & 0.01 & 9 \\
KSIP & 2 & 0.08 & 0.73 & 1.53 & 2 & 0.01 & 0.74 & 1.70 & 11 \\
MOSARQP1 & 2 & 0.05 & 0.04 & 0.11 & 2 & 0.02 & 0.04 & 0.07 & 15 \\
PRIMALC1 & 0 & 0.06 & 4.54 & 4.60 & 0 & 0.00 & 1.17 & 1.17 & 15 \\
PRIMALC2 & 0 & 0.00 & 4.22 & 4.23 & 0 & 0.00 & 1.01 & 1.01 & 15 \\
PRIMALC5 & 0 & 0.01 & 6.84 & 6.85 & 0 & 0.00 & 1.62 & 1.62 & 15 \\
PRIMALC8 & 0 & 0.01 & 28.59 & 28.60 & 0 & 0.00 & 5.15 & 5.16 & 15 \\
\hline STOKES1 & 52 & 25.97 & 50.53 & 76.51 & 52 & 4.09 & 0.58 & 4.68 & 2 \\
STOKES2 & - & - & - & - & 187 & 237.68 & 11.64 & 249.41 & - \\
STOKES3 & 45 & 453.50 & 374.39 & 827.94 & 46 & 36.96 & 1.61 & 38.65 & 2 \\
STOKES4 & - & - & - & - & - & - & - & - & - \\
STOKES5 & 35 & 182.53 & 179.95 & 362.63 & 34 & 8.40 & 1.53 & 10.08 & 3 \\
STOKES6 & - & - & - & - & - & - & - & - & - \\
STOKES7 & 8 & 15.63 & 6.25 & 21.89 & 8 & 1.47 & 0.06 & 1.53 & 14 \\
STOKES8 & 11 & 943.87 & 167.57 & 1111.47 & 11 & 27.20 & 0.36 & 27.58 & 13
\end{tabular}

\begin{tabular}{l|rrr|rrr} 
& \multicolumn{3}{|c}{$\mathrm{LU}$} & \multicolumn{3}{c}{ Schilders' } \\
Problem & $\mathrm{nz}(L(\mathcal{P}))$ & $\mathrm{nz}(U(\mathcal{P}))$ & Total & $\mathrm{nz}\left(L\left(D_{2}\right)\right)$ & $\mathrm{nz}\left(U\left(D_{2}\right)\right)$ & Total \\
\hline CONT-050 & 595768 & 649016 & 1244784 & 196 & 196 & 117085 \\
CONT-101 & 3681821 & 2776506 & 6458327 & 99 & 99 & 683747 \\
CONT-300 & - & - & - & - & - & - \\
CONT1-10 & 4867901 & 5674469 & 10542370 & 396 & 396 & 677437 \\
CVXQP1 & 25787124 & 39299255 & 65086379 & 5000 & 5000 & 91004 \\
CVXQP2 & 8243264 & 10825817 & 19069081 & 7500 & 7500 & 43683 \\
CVXQP3 & 24645462 & 44499396 & 69144858 & 2500 & 2500 & 113266 \\
DUAL1 & 3604 & 3634 & 7238 & 84 & 84 & 337 \\
DUAL2 & 4579 & 4544 & 9123 & 95 & 95 & 377 \\
DUAL3 & 6140 & 6103 & 12243 & 110 & 110 & 441 \\
DUAL4 & 2882 & 2805 & 5687 & 74 & 74 & 297 \\
KSIP & 22024 & 22024 & 44048 & 20 & 20 & 22044 \\
MOSARQP1 & 19264 & 19276 & 38540 & 2500 & 2500 & 10181 \\
PRIMALC1 & 248 & 4387 & 4635 & 230 & 229 & 2547 \\
PRIMALC2 & 245 & 3478 & 3723 & 231 & 230 & 2092 \\
PRIMALC5 & 303 & 4894 & 5197 & 287 & 286 & 2885 \\
PRIMALC8 & 536 & 8855 & 9391 & 520 & 519 & 5215 \\
\hline STOKES1 & 1684985 & 2104142 & 3789127 & 1890 & 1890 & 19875 \\
STOKES2 & - & - & - & 7362 & 7362 & 87160 \\
STOKES3 & 13281099 & 16195569 & 29476668 & 5121 & 5121 & 55944 \\
STOKES4 & - & - & - & - & - & - \\
STOKES5 & 7621013 & 13040444 & 20661457 & 3778 & 3778 & 62434 \\
STOKES6 & - & - & - & - & - & - \\
STOKES7 & 1308824 & 1706168 & 3014992 & 1923 & 1923 & 11678 \\
STOKES8 & 23764396 & 31623689 & 55388085 & 7427 & 7427 & 48126
\end{tabular}


TABLE 5.5

Solution statistics: Comparisons between iterative PCG solvers when using lu and Schilders' factorizations in the preconditioning step with tol $=10^{-2}, G_{1,1}=A_{1,1}, G_{1,2}=G_{2,1}^{T}=A_{1,2}$, and $D_{2}=A_{2,2}$.

\begin{tabular}{|c|c|c|c|c|c|c|c|c|c|}
\hline \multirow[b]{2}{*}{ Problem } & \multicolumn{4}{|c|}{$\mathrm{LU}$} & \multicolumn{4}{|c|}{ Schilders' } & \multirow[b]{2}{*}{ Digits } \\
\hline & Its & FTime & ITime & Total & Its & FTime & ITime & Total & \\
\hline CONT-050 & 1 & 8.35 & 0.66 & 9.12 & 1 & 0.06 & 0.45 & 0.60 & 11 \\
\hline CONT-101 & 1 & 41.44 & 4.50 & 46.71 & 1 & 0.45 & 2.84 & 4.06 & 6 \\
\hline CONT-300 & - & - & - & - & 1 & 14.48 & 60.19 & 98.69 & - \\
\hline CONT1-10 & 1 & 147.41 & 5.66 & 153.84 & 1 & 0.44 & 2.89 & 4.11 & 12 \\
\hline CVXQP1 & 401 & 952.69 & 9531.04 & 10483.85 & 134 & 0.09 & 15.05 & 15.24 & 3 \\
\hline CVXQP2 & 0 & 226.36 & 10.12 & 236.50 & 1 & 1.80 & 1.50 & 3.32 & 0 \\
\hline CVXQP3 & 29 & 587.99 & 522.23 & 1110.56 & 18 & 0.08 & 2.77 & 3.19 & 1 \\
\hline DUAL1 & 1 & 0.01 & 0.00 & 0.01 & 1 & 0.00 & 0.00 & 0.01 & 11 \\
\hline DUAL2 & 1 & 0.02 & 0.00 & 0.02 & 1 & 0.01 & 0.00 & 0.01 & 12 \\
\hline DUAL3 & 2 & 0.02 & 0.01 & 0.03 & 2 & 0.01 & 0.00 & 0.01 & 9 \\
\hline DUAL4 & 2 & 0.01 & 0.00 & 0.01 & 2 & 0.00 & 0.00 & 0.01 & 10 \\
\hline KSIP & 0 & 0.07 & 6.60 & 7.40 & 0 & 0.00 & 1.59 & 2.31 & 15 \\
\hline MOSARQP1 & 0 & 0.04 & 172.38 & 172.44 & 0 & 0.01 & 142.10 & 142.13 & 15 \\
\hline PRIMALC1 & 0 & 0.01 & 4.24 & 4.25 & 0 & 0.00 & 1.14 & 1.15 & 15 \\
\hline PRIMALC2 & 0 & 0.00 & 4.00 & 4.01 & 0 & 0.00 & 1.00 & 1.00 & 15 \\
\hline PRIMALC5 & 0 & 0.01 & 6.55 & 6.55 & 0 & 0.00 & 1.71 & 1.71 & 15 \\
\hline PRIMALC8 & 0 & 0.01 & 28.06 & 28.07 & 0 & 0.00 & 5.10 & 5.10 & 15 \\
\hline STOKES1 & 91 & 23.21 & 91.12 & 114.35 & 92 & 0.05 & 1.22 & 1.28 & 2 \\
\hline STOKES2 & - & - & - & - & 560 & 0.31 & 39.42 & 39.82 & - \\
\hline STOKES3 & 79 & 450.28 & 688.39 & 1138.72 & 75 & 0.17 & 3.17 & 3.39 & 3 \\
\hline STOKES4 & - & - & - & - & 234 & 1.02 & 48.17 & 49.47 & - \\
\hline STOKES5 & 70 & 174.76 & 364.39 & 539.31 & 63 & 0.13 & 3.03 & 3.31 & 3 \\
\hline STOKES6 & - & - & - & - & 136 & 0.79 & 28.75 & 30.59 & - \\
\hline STOKES7 & 14 & 14.35 & 11.17 & 25.53 & 14 & 0.05 & 0.14 & 0.20 & 11 \\
\hline STOKES8 & 28 & 834.47 & 434.78 & 1269.28 & 28 & 0.28 & 1.25 & 1.55 & 7 \\
\hline
\end{tabular}

\begin{tabular}{|c|c|c|c|c|c|c|}
\hline Problem & $\mathrm{nz}(L(\mathcal{P}))$ & $\begin{array}{c}\mathrm{LU} \\
\mathrm{nz}(U(\mathcal{P}))\end{array}$ & Total & $\mathrm{nz}\left(L\left(D_{2}\right)\right)$ & $\begin{array}{l}\text { Schilders' } \\
\mathrm{nz}\left(U\left(D_{2}\right)\right)\end{array}$ & Total \\
\hline CONT-050 & 595768 & 648968 & 1244736 & 196 & 196 & 117085 \\
\hline CONT-101 & 3690797 & 2775736 & 6466533 & 99 & 99 & 683747 \\
\hline CONT-300 & - & - & - & 299 & 299 & 10794780 \\
\hline CONT1-10 & 4867901 & 5800598 & 10668499 & 396 & 396 & 677437 \\
\hline CVXQP1 & 31909093 & 44477738 & 76386831 & 23310 & 23734 & 128048 \\
\hline CVXQP2 & 14908190 & 16628011 & 31536201 & 847610 & 572256 & 1448549 \\
\hline CVXQP3 & 24497857 & 41965765 & 66463622 & 4926 & 4926 & 118118 \\
\hline DUAL1 & 3739 & 3739 & 7478 & 3568 & 3568 & 7305 \\
\hline DUAL2 & 4747 & 4747 & 9494 & 4554 & 4554 & 9295 \\
\hline DUAL3 & 6326 & 6326 & 12652 & 6104 & 6104 & 12429 \\
\hline DUAL4 & 2924 & 2924 & 5848 & 2775 & 2775 & 5699 \\
\hline KSIP & 22024 & 22023 & 44047 & 20 & 19 & 22043 \\
\hline MOSARQP1 & 9821 & 8304 & 18125 & 2536 & 2186 & 9903 \\
\hline PRIMALC1 & 248 & 4387 & 4635 & 230 & 229 & 2547 \\
\hline PRIMALC2 & 245 & 3478 & 3723 & 231 & 230 & 2092 \\
\hline PRIMALC5 & 303 & 4894 & 5197 & 287 & 286 & 2885 \\
\hline PRIMALC8 & 536 & 8855 & 9391 & 520 & 519 & 5215 \\
\hline STOKES1 & 1684987 & 2104662 & 3789649 & 28475 & 28475 & 73045 \\
\hline STOKES2 & - & - & - & 190490 & 190490 & 453416 \\
\hline STOKES3 & 13362756 & 16195611 & 29558367 & 99430 & 99430 & 244562 \\
\hline STOKES4 & - & - & - & 620644 & 620715 & 1439851 \\
\hline STOKES5 & 7617212 & 13057170 & 20674382 & 79728 & 79728 & 214334 \\
\hline STOKES6 & - & - & - & 439216 & 452489 & 1122908 \\
\hline STOKES7 & 1314702 & 1707861 & 3022563 & 29666 & 29666 & 67164 \\
\hline STOKES8 & 23715838 & 31936839 & 55652677 & 177137 & 177137 & 387546 \\
\hline
\end{tabular}


TABLE 5.6

Solution statistics: Comparisons between iterative PCG solvers when using lu and Schilders' factorizations in the preconditioning step with tol $=10^{-2}, G_{1,1}=0, G_{1,2}=0$, and $D_{2}=A_{2,2}$.

\begin{tabular}{|c|c|c|c|c|c|c|c|c|c|}
\hline \multirow[b]{2}{*}{ Problem } & \multicolumn{4}{|c|}{$\mathrm{LU}$} & \multicolumn{4}{|c|}{ Schilders' } & \multirow[b]{2}{*}{ Digits } \\
\hline & Its & FTime & ITime & Total & Its & FTime & ITime & Total & \\
\hline CONT-050 & 1 & 0.72 & 0.38 & 1.20 & 1 & 0.06 & 0.43 & 0.59 & 11 \\
\hline CONT-101 & 1 & 5.39 & 2.50 & 8.66 & 1 & 0.46 & 2.85 & 4.09 & 10 \\
\hline CONT-300 & 1 & 324.00 & 98.27 & 446.28 & 1 & 14.59 & 59.78 & 98.46 & 14 \\
\hline CONT1-10 & 1 & 8.24 & 3.00 & 12.05 & 1 & 0.45 & 2.84 & 4.07 & 10 \\
\hline CVXQP1 & 119 & 29.27 & 330.01 & 359.39 & 104 & 0.09 & 10.20 & 10.39 & 2 \\
\hline CVXQP2 & 3 & 7.73 & 4.53 & 12.28 & 0 & 1.78 & 0.99 & 2.79 & 0 \\
\hline CVXQP3 & 16 & 7.21 & 18.37 & 25.91 & 16 & 0.07 & 2.11 & 2.52 & 4 \\
\hline DUAL1 & 1 & 0.01 & 0.00 & 0.01 & 1 & 0.00 & 0.00 & 0.01 & 15 \\
\hline DUAL2 & 1 & 0.01 & 0.00 & 0.02 & 1 & 0.01 & 0.00 & 0.01 & 15 \\
\hline DUAL3 & 2 & 0.02 & 0.00 & 0.02 & 2 & 0.01 & 0.00 & 0.01 & 14 \\
\hline DUAL4 & 2 & 0.01 & 0.00 & 0.01 & 2 & 0.00 & 0.00 & 0.01 & 12 \\
\hline KSIP & 0 & 0.34 & 6.67 & 7.74 & 0 & 0.00 & 1.59 & 2.32 & 15 \\
\hline MOSARQP1 & 0 & 0.03 & 149.10 & 149.14 & 0 & 0.01 & 136.80 & 136.82 & 15 \\
\hline PRIMALC1 & 0 & 0.01 & 4.35 & 4.36 & 0 & 0.00 & 1.15 & 1.15 & 15 \\
\hline PRIMALC2 & 0 & 0.00 & 4.03 & 4.03 & 0 & 0.00 & 1.01 & 1.01 & 15 \\
\hline PRIMALC5 & 0 & 0.01 & 6.85 & 6.85 & 0 & 0.00 & 1.62 & 1.62 & 15 \\
\hline PRIMALC8 & 0 & 0.01 & 28.28 & 28.29 & 0 & 0.00 & 5.18 & 5.18 & 15 \\
\hline STOKES1 & 75 & 0.21 & 3.05 & 3.28 & 79 & 0.05 & 1.02 & 1.08 & 2 \\
\hline STOKES2 & 240 & 4.75 & 259.71 & 264.55 & 254 & 0.30 & 16.79 & 17.18 & 2 \\
\hline STOKES3 & 65 & 0.81 & 8.52 & 9.38 & 65 & 0.16 & 2.59 & 2.80 & 3 \\
\hline STOKES4 & 179 & 9.79 & 211.73 & 221.81 & 163 & 0.98 & 31.93 & 33.21 & 5 \\
\hline STOKES5 & 58 & 1.48 & 20.53 & 22.15 & 59 & 0.12 & 2.51 & 2.78 & 4 \\
\hline STOKES6 & 133 & 45.24 & 543.96 & 590.21 & 128 & 0.69 & 23.81 & 25.49 & 2 \\
\hline STOKES7 & 14 & 0.10 & 0.31 & 0.41 & 14 & 0.05 & 0.13 & 0.18 & 14 \\
\hline STOKES8 & 28 & 0.70 & 4.05 & 4.77 & 28 & 0.27 & 1.15 & 1.45 & 10 \\
\hline
\end{tabular}

\begin{tabular}{l|rrr|rrr} 
& \multicolumn{3}{|c}{ LU } & \multicolumn{3}{|c}{ Schilders' } \\
Problem & $\mathrm{nz}(L(\mathcal{P}))$ & $\mathrm{nz}(U(\mathcal{P}))$ & Total & $\mathrm{nz}\left(L\left(D_{2}\right)\right)$ & $\mathrm{nz}\left(U\left(D_{2}\right)\right)$ & Total \\
\hline CONT-050 & 293011 & 411916 & 704927 & 196 & 196 & 114684 \\
CONT-101 & 1770989 & 2230310 & 4001299 & 99 & 99 & 681146 \\
CONT-300 & 45690125 & 62556439 & 108246564 & 299 & 299 & 10704482 \\
CONT1-10 & 2194946 & 3280161 & 5475107 & 396 & 396 & 667636 \\
CVXQP1 & 4346056 & 3841931 & 8187987 & 23310 & 23734 & 89800 \\
CVXQP2 & 1856579 & 1343030 & 3199609 & 847610 & 572256 & 1430081 \\
CVXQP3 & 1831354 & 1842046 & 3673400 & 4926 & 4926 & 63978 \\
DUAL1 & 3570 & 3738 & 7308 & 3568 & 3568 & 7222 \\
DUAL2 & 4556 & 4746 & 9302 & 4554 & 4554 & 9205 \\
DUAL3 & 6106 & 6326 & 12432 & 6104 & 6104 & 12320 \\
DUAL4 & 2777 & 2925 & 5702 & 2775 & 2775 & 5626 \\
KSIP & 22022 & 22023 & 44045 & 20 & 19 & 22042 \\
MOSARQP1 & 7325 & 7066 & 14391 & 2536 & 2186 & 9544 \\
PRIMALC1 & 248 & 4387 & 4635 & 230 & 229 & 2547 \\
PRIMALC2 & 245 & 3478 & 3723 & 231 & 230 & 2092 \\
PRIMALC5 & 303 & 4894 & 5197 & 287 & 286 & 2885 \\
PRIMALC8 & 536 & 8855 & 9391 & 520 & 519 & 5215 \\
\hline STOKES1 & 83694 & 181989 & 265683 & 28475 & 28475 & 69959 \\
STOKES2 & 2329914 & 1548050 & 3877964 & 190490 & 190490 & 441945 \\
STOKES3 & 258848 & 797838 & 1056686 & 99430 & 99430 & 236513 \\
STOKES4 & 2486613 & 6207846 & 8694459 & 620644 & 620715 & 1409233 \\
STOKES5 & 768367 & 542274 & 1310641 & 79728 & 79728 & 192276 \\
STOKES6 & 8697371 & 6909772 & 15607143 & 439216 & 452489 & 1032170 \\
STOKES7 & 50998 & 58625 & 109623 & 29666 & 29666 & 63539 \\
STOKES8 & 340761 & 379503 & 720264 & 177137 & 177137 & 372599
\end{tabular}


TABLE 5.7

Solution statistics: Comparison of different preconditioners with the preferable factorization method and tol $=10^{-6}$. (a) $G=I$, (b) $G=\operatorname{diag}(A)$, (c) $G_{1,1}=A_{1,1}, G_{1,2}=G_{2,1}^{T}=A_{1,2}$, and $D_{2}=A_{2,2}$, (d) $G_{1,1}=0, G_{1,2}=G_{2,1}^{T}=0$, and $D_{2}=A_{2,2}$.

\begin{tabular}{l|rr|rr|rr|rr} 
& \multicolumn{2}{|c|}{ LU (a) } & \multicolumn{2}{|c|}{ LU (b) } & \multicolumn{2}{|c|}{ Schilders' (c) } & \multicolumn{2}{|c}{ Schilders' (d) } \\
Name & Its & Time & Its & Time & Its & Time & Its & Time \\
\hline CONT-050 & 3 & 2.68 & 1 & 2.06 & 4 & 1.13 & 4 & 1.09 \\
CONT-101 & 7 & 45.98 & 1 & 16.21 & 2 & 5.14 & 2 & 5.11 \\
CONT-300 & - & - & - & - & 4 & 155.35 & 4 & 153.56 \\
CONT1-10 & 1 & 27.66 & 1 & 26.17 & 4 & 7.36 & 4 & 7.12 \\
CVXQP1 & 16 & 9.04 & 9 & 5.98 & 773 & 85.96 & 601 & 58.43 \\
CVXQP2 & 18 & 0.72 & 9 & 0.41 & 1 & 3.44 & 0 & 2.81 \\
CVXQP3 & 12 & 15.93 & 6 & 11.35 & 161 & 21.60 & 141 & 15.79 \\
DUAL1 & 38 & 0.02 & 37 & 0.02 & 3 & 0.01 & 3 & 0.01 \\
DUAL2 & 22 & 0.02 & 21 & 0.02 & 4 & 0.02 & 3 & 0.02 \\
DUAL3 & 24 & 0.03 & 24 & 0.03 & 2 & 0.02 & 2 & 0.02 \\
DUAL4 & 14 & 0.01 & 13 & 0.01 & 3 & 0.01 & 3 & 0.01 \\
KSIP & 20 & 2.13 & 1 & 1.93 & 0 & 2.33 & 0 & 2.28 \\
MOSARQP1 & 21 & 0.28 & 5 & 0.08 & 0 & 142.22 & 0 & 136.41 \\
PRIMALC1 & 6 & 1.34 & 0 & 4.36 & 0 & 1.16 & 0 & 1.14 \\
PRIMALC2 & 2 & 1.27 & 0 & 4.02 & 0 & 1.01 & 0 & 1.00 \\
PRIMALC5 & 0 & 2.13 & 0 & 6.59 & 0 & 1.64 & 0 & 1.64 \\
PRIMALC8 & 2 & 7.88 & 0 & 28.52 & 0 & 5.17 & 0 & 5.10 \\
\hline STOKES1 & 36 & 0.32 & 32 & 0.30 & 298 & 4.08 & 257 & 3.21 \\
STOKES2 & 67 & 2.50 & 57 & 2.21 & 3275 & 230.97 & 1575 & 104.44 \\
STOKES3 & 23 & 0.90 & 21 & 0.84 & 397 & 16.66 & 330 & 13.08 \\
STOKES4 & 38 & 5.06 & 34 & 4.66 & 2093 & 425.20 & 1460 & 283.37 \\
STOKES5 & 23 & 1.45 & 21 & 1.36 & 333 & 15.56 & 309 & 12.42 \\
STOKES6 & 37 & 10.90 & 35 & 10.38 & 1382 & 281.50 & 1261 & 226.31 \\
STOKES7 & 35 & 0.28 & 23 & 0.20 & 45 & 0.49 & 45 & 0.44 \\
STOKES8 & 77 & 2.91 & 38 & 1.49 & 116 & 5.33 & 115 & 4.73
\end{tabular}

The number of iterations carried out is given in the column headed "Its." We also measure the CPU time taken in carrying out the factorization of the preconditioners (FTime) and the CPU time then taken by Algorithm 3.2 when using these factored forms (ITime). The time spent finding an initial $x$ which satisfies $B x=d$ is excluded from the value given in Itime. The total CPU time used to solve the problem is given by "Total." Each problem is solved ten times and the mean of the CPU times recorded. The minimum values of $\frac{1}{2} x^{T} A x+s^{T} x$ calculated are also compared for all the problems, although this is not immediately applicable to the Stokes problems. The number of digits of agreement in these values are given in "Digits." We observe that for the majority of the CUTEr problems, the differences in rounding errors of the two factorization methods do not greatly affect the calculated minimum values. We note that for some of the Stokes problems at least one of the solutions is rather inaccurate, though we have no easy way of finding out which! An important factor in how well a certain factorization will perform is its sparsity; in particular, the number of nonzeros in the $\mathrm{lu}$ factors of $\mathcal{P}$, and the number of nonzeros in the factors of $D_{2}$. These values are also given for each of our preconditioners, and the total amount of storage required for the various blocks required. Note that the matrices $D_{1}$ and $E$ should not be generated when using Schilders' factorization.

The diagonal form of $D_{2}$ used in the examples of Table 5.4 produces a significant 


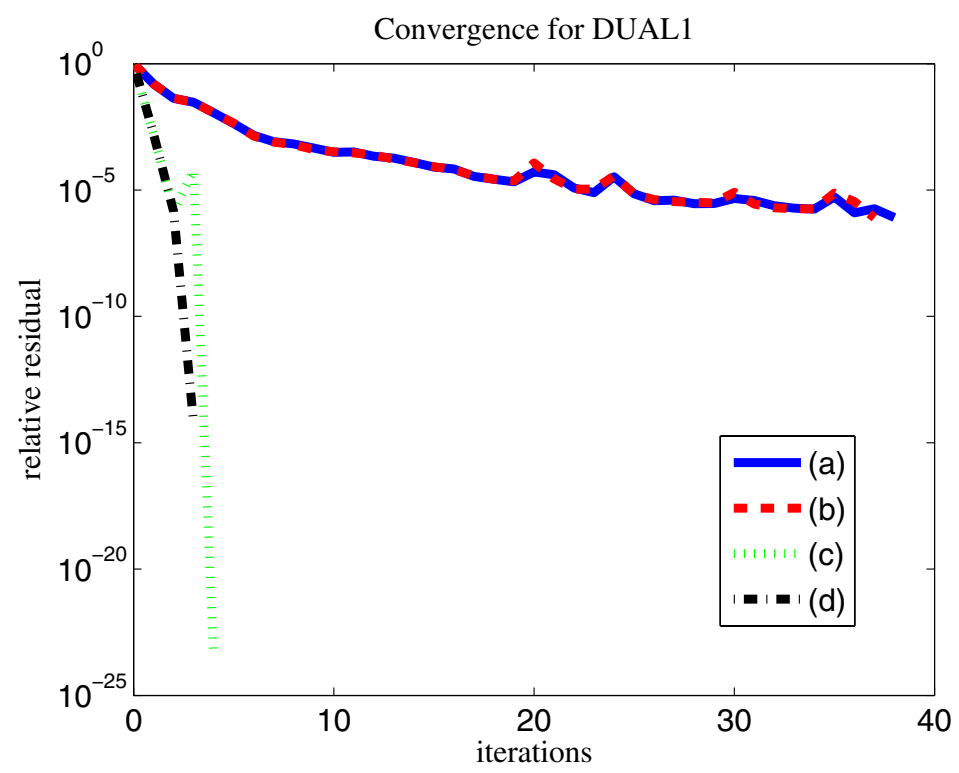

FIG. 5.1. Convergence of relative residuals $r^{T} g$ with the different preconditioning strategies from Table 5.7 for the problem DUAL1.

drop in the CPU times when we use the Schilders' factorization instead of the lu factorization of $\mathcal{P}$. However, this choice of $D_{2}$ is not so practical because we have to form the matrix $\widehat{D}_{2}$, as defined in (4.6), and then use the diagonal. If $A_{2,2}$ is positive definite and sparse enough for us to consider factorizing it, then we can set $D_{2}=A_{2,2}$ with $G_{1,1}=A_{1,1}$ and $G_{1,2}=G_{2,1}^{T}=A_{1,2}$. From Theorem 4.4 we know that the dimension of the Krylov subspace $\mathcal{K}\left(\mathcal{P}^{-1} \mathcal{A}, b\right)$ is at most $\min (n-m+1,2 m+2)$. The results of using such a preconditioner are shown in Table 5.5. In particular, note the four DUAL* problems. For each of these $m=1$, so the upper bound on the dimension of the Krylov subspace $\mathcal{K}\left(\mathcal{P}^{-1} \mathcal{A}, b\right)$ is at most 4 . Our results support this claim.

All tests were performed on a dual Intel Xeon $3.20 \mathrm{GHz}$ machine with hyperthreading and 2GB of RAM. It was running Fedora Core 2 (Linux kernel 2.6.8) and all programs were written in MATLAB 7.0. We terminate the iteration when the value of $r^{T} g$ is reduced by at least a factor of $10^{-2}$. If no result is given for a problem, then the machine we used ran out of memory during the factorization stage. This is indicated by - in the results tables. Consider the test problem CONT-300. We were unable to solve it by choosing $G$ explicitly, as in Tables 5.2 and 5.3, and then factoring it because of the machine running out of memory. However, when we used Schilders' factorization with $D_{2}=A_{2,2}$, our algorithm successfully terminated, as in Tables 5.5 and 5.6. We were also able to factor $\mathcal{P}$ with an $\mathrm{lu}$ factorization for $G_{1,1}=0$, $G_{1,2}=G_{2,1}^{T}=0$, and $D_{2}=A_{2,2}$, but the factorization time was over 300 times larger than using Schilders' factorization, resulting in the total CPU time being at least 38 times larger.

It is clear that, for the majority of the problems, factoring $\mathcal{P}$ with an lu factorization is preferable when we choose $G$ as in Tables 5.2 and 5.3. However, it is generally preferable to use Schilders' factorization for the other choices of preconditioner, as we would expect. Table 5.7 shows results for the factorization of preference with four of the preconditioners that we have considered. The iteration process is terminated 
when $r^{T} g$ has been reduced by a factor of $10^{-6}$. The number of iterations required and the total CPU time is recorded. We observe that Schilders' factorization with $G_{1,1}=0, G_{1,2}=0$, and $D_{2}=A_{2,2}$ is preferable, or of equal preference, for twelve out of the seventeen CUTEr test problems. However, setting $G=\operatorname{diag}(A)$ and then carrying out an $\mathrm{lu}$ factorization is better for the Stokes problems tested because the number of iterations required is greatly reduced. The convergence of the relative residuals of $r^{T} g$ are plotted in Figure 5.1 for the problem DUAL1. The line labeled "(c)" corresponds to a preconditioner which satisfies the assumptions of Theorem 4.4. The plot supports this theorem.

6. Conclusion. In this paper, we have discussed the use of the PCG method for solving indefinite linear systems. We have investigated the use of a new factorization for constraint preconditioners and shown how this can be used in incomplete forms to decrease the number of flops required overall by the preconditioned iterative method. We obtained an upper bound on the number of iterations required to solve systems of the form (1.1) by means of appropriate Krylov subspace methods by using a minimum polynomial argument.

We have provided computational evidence that the PCG method works well for nontrivial quadratic programming problems; however, it would not be the method of choice for the Stokes saddle-point problems tested. Indeed, problem-based preconditioned iterative methods which have optimal computational complexity exist for these problems (see [2, Chapter 6]).

In some cases, the use of Schilders' factorization to define a preconditioner can speed up the solution time by a factor of 5 compared to using the lu factorization of a preconditioner for which $G$ has been chosen. For larger dimensional problems, we have shown an example where Schilders' factorization approach enables fast solution but the $\mathrm{lu}$ factorization with popular choices of preconditioner fails because of memory costs.

The cost of finding $m$ linearly independent columns in the matrix $B$ via a permuted $\mathrm{lu}$ factorization is often higher than we would like. We plan to investigate different strategies for this in the future, and how these may change the number of iterations required to solve the problems.

Acknowledgments. The authors would like to thank Nick Gould and Wil Schilders for their helpful input during the early stages of this research.

\section{REFERENCES}

[1] L. Bergamaschi, J. Gondzio, and G. Zilli, Preconditioning indefinite systems in interior point methods for optimization, Comput. Optim. Appl., 28 (2004), pp. 149-171.

[2] H. C. Elman, D. J. Silvester, And A. J. Wathen, Finite Elements and Fast Iterative Solvers: With Applications in Incompressible Fluid Dynamics, Oxford University Press, Oxford, UK, to appear.

[3] B. Fischer, Polynomial Based Iteration Methods for Symmetric Linear Systems, Adv. Numer. Math., John Wiley \& Sons Ltd., Chichester, UK; B. G. Teubner, Stuttgart, Germany, 1996.

[4] P. E. Gill, W. Murray, and M. H. Wright, Practical Optimization, Academic Press Inc. [Harcourt Brace Jovanovich Publishers], London, 1981.

[5] G. H. Golub and C. F. Van Loan, Matrix Computations, 3rd ed., Johns Hopkins Stud. Math. Sci., Johns Hopkins University Press, Baltimore, MD, 1996.

[6] N. I. M. Gould, D. Orban, And Ph. L. Toint, CUTEr (and SifDec), a Constrained and Unconstrained Testing Environment, Revisited, Tech. Report TR/PA/01/04, CERFACS, Toulouse, France, 2001. 
[7] N. I. M. Gould, M. E. Hribar, And J. Nocedal, On the solution of equality constrained quadratic programming problems arising in optimization, SIAM J. Sci. Comput., 23 (2001), pp. $1376-1395$.

[8] C. Keller, N. I. M. Gould, and A. J. Wathen, Constraint preconditioning for indefinite linear systems, SIAM J. Matrix Anal. Appl., 21 (2000), pp. 1300-1317.

[9] L. LUKŠAN AND J. VLČEK, Indefinitely preconditioned inexact Newton method for large sparse equality constrained non-linear programming problems, Numer. Linear Algebra Appl., 5 (1998), pp. 219-247.

[10] A. Quarteroni and A. Valli, Numerical Approximation of Partial Differential Equations, Springer Series in Computational Mathematics 23, Springer-Verlag, Berlin, 1994.

[11] W. SCHILDERS, private communication, 2002.

[12] H. A. VAn DER Vorst, Iterative Krylov Methods for Large Linear Systems, Cambridge Monographs on Applied and Computationl Mathematics, Cambridge University Press, Cambridge, UK, 2003.

[13] S. J. Wright, Primal-Dual Interior-Point Methods, SIAM, Philadelphia, PA, 1997. 